\title{
PARTICLE DISTRIBUTIONS FOR BEAM IN ELECTRIC FIELD*
}

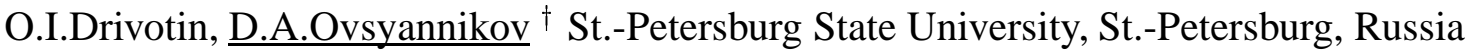

\section{Abstract}

In the present report an approach for determination of particle distribution density in analytical form for beam in electric field is presented. It was applied previously for beams in magnetic fields [1]. The purpose of the report is to extend this approach on beams in electric field. For low current beam, the expressions for particle density are obtained in various cases. For intense beam, the integral equation for particle density in the space of first integrals of motion equations is proposed. The well known KV distribution is one of its solutions.

\section{ERMAKOV SYSTEMS AND THEIR INTEGRALS}

Consider stationary beam of charged particles in RF electric field, transverse components of which $E_{x}, E_{y}$ are linear functions of the corresponding coordinates:

$$
E_{x}=k_{x} x, \quad E_{y}=k_{y} y .
$$

Let assume that in each cross-section of the beam all particles have the same longitudinal velocity $\dot{z}$. This assumption is realized, for example, for beam in initial part of RFQ channel.

Further we will consider distribution of particles of some infinitely thin layer moving along $z$-axis with the velocity $\dot{z}$ and restricted by two infinitely closed planes moving with the same velocity. Let us assume that initially (in the beginning of the channel) particles fill ellipsoid in fourdimensional space:

$$
X^{*} B_{0} X=1, \quad X^{*}=(x, \dot{x}, y, \dot{y}),
$$

$B_{0}$ is diagonal matrix, $B_{0}=\operatorname{diag}\left(a_{x 0}, c_{x 0}, a_{y 0}, c_{y 0}\right)$. As the equations of motion are linear:

$$
\frac{d^{2} x}{d t^{2}}=Q_{x} x, \quad \frac{d^{2} y}{d t^{2}}=Q_{y} y, \quad Q_{x, y}=k_{x, y} / m
$$

in each subsequent moment particles fill ellipsoid, and envelopes on $x, y$ satisfy the equations

$$
\begin{aligned}
\frac{d^{2} R_{x}}{d t^{2}} & =Q_{x} R_{x}+\frac{E_{x}^{2}}{R_{x}^{3}} \\
\frac{d^{2} R_{y}}{d t^{2}} & =Q_{y} R_{y}+\frac{E_{y}^{2}}{R_{y}^{3}},
\end{aligned}
$$

where $E_{x}=a_{x 0} c_{x 0}, E_{y}=a_{y 0} c_{y 0}$. Here $R_{x, y}$ are beam envelopes. The equations (3) and (4,5) represent two pairs

\footnotetext{
* This work is supported by Russian Foundation for Fundamental Researches, project 99-01-00678

† E-mail:Dmitri.Ovsyannikov@pobox.spbu.ru
}

of Ermakov systems [2]. They have well known Ermakov integrals

$$
\begin{gathered}
I_{x}^{2}=\left(x \dot{R}_{x}-\dot{x} R_{x}\right)^{2}+\frac{E_{x}^{2}}{R_{x}^{2}} x^{2}, \\
I_{y}^{2}=\left(y \dot{R}_{y}-\dot{y} R_{y}\right)^{2}+\frac{E_{y}^{2}}{R_{y}^{2}} y^{2} .
\end{gathered}
$$

Assume that the beam cross section is bounded by the ellipse:

$$
x^{2} / R_{x}^{2}+y^{2} / R_{y}^{2} \leq 1,
$$

and find such set of $I_{x}$ and $I_{y}$ that this assumption would be true. Maximal value of the coordinate $x$ is reached when the first term in (6) turns to zero:

$$
x_{\max }^{2}=I_{x}^{2} R_{x}^{2} / E_{x}^{2} .
$$

Similarly,

$$
y_{\max }^{2}=I_{y}^{2} R_{y}^{2} / E_{y}^{2} .
$$

The point $\left(x_{\max }, y_{\max }\right)$ must lie inside the ellipse $x^{2} / R_{x}^{2}+$ $y^{2} / R_{y}^{2}=1$ and we get

$$
\frac{I_{x}^{2}}{E_{x}^{2}}+\frac{I_{y}^{2}}{E_{y}^{2}} \leq 1, \quad I_{x} \geq 0, \quad I_{y} \geq 0 .
$$

So, the set of admissible values of $I_{x}, I_{y}$ is bounded by the ellipse (9) and coordinate axes.

\section{DISTRIBUTIONS IN THE SPACE OF INTEGRALS}

Let us consider particle distribution of the moving infinitely thin layer introduced above. Taking into account that the thickness of the layer $d z$ varies when the layer moves along $z$-axis, we normalize all densities dividing them by conserving value $d z / \dot{z}$.

We will denote the density of distribution on the variables $a, b, \ldots$ by $D N / D(a, b, \ldots)$. For example, the phase density in this notation is $n=D N / D(x, \dot{x}, y, \dot{y})$. Assume that the phase density depends only on values of the integrals $I_{x}$ and $I_{y}$. Under this condition the particle distribution is determined only by $I_{x}$ and $I_{y}$, and, hence, we can introduce the density of distribution on values of the integrals $I_{x}$ and $I_{y} f\left(I_{x}, I_{y}\right)=D N / D\left(I_{x}, I_{y}\right)$. Note, that setting of $f\left(I_{x}, I_{y}\right)$ as function of $I_{x}, I_{y}$ is more correct procedure then setting of phase density $n$ as function of $I_{x}, I_{y}$ because we set density as function of the variables to which it is related as a density. In particular, we can correctly use generalized function as particle densities.

Let us express the phase density and the density in the configuration space through the density $f\left(I_{x}, I_{y}\right)$. Introduce the variables

$$
q_{x}=x / R_{x}, \quad q_{y}=y / R_{y}
$$




$$
s_{x}=\left(x \dot{R}_{x}-\dot{x} R_{x}\right), \quad s_{y}=\left(y \dot{R}_{y}-\dot{y} R_{y}\right) .
$$

Then we have

$$
\begin{gathered}
\frac{D N}{D\left(q_{x}, q_{y}, I_{x}, I_{y}\right)}=4 \frac{D N}{D\left(q_{x}, q_{y}, s_{x}, s_{y}\right)}\left|\operatorname{det} \frac{\partial\left(s_{x}, s_{y}\right)}{\partial\left(I_{x}, I_{y}\right)}\right|= \\
\frac{4 I_{x} I_{y}}{\left|s_{x} s_{y}\right|} \cdot \frac{D N}{D\left(q_{x}, q_{y}, s_{x}, s_{y}\right)}=\frac{4 I_{x} I_{y}}{\left|s_{x} s_{y}\right|} \cdot \frac{1}{R_{x}^{2} R_{y}^{2}} \times \\
\frac{D N}{D\left(q_{x}, q_{y}, \dot{q}_{x}, \dot{q}_{y}\right)}=\frac{4 I_{x} I_{y}}{\left|s_{x} s_{y}\right|} \cdot \frac{1}{R_{x}^{2} R_{y}^{2}} \cdot \frac{D N}{D(x, y, \dot{x}, \dot{y})} \times \\
\left|\operatorname{det} \frac{\partial(x, y, \dot{x}, \dot{y})}{\partial\left(q_{x}, q_{y}, \dot{q}_{x}, \dot{q}_{y}\right)}\right|=\frac{4 I_{x} I_{y}}{\left|s_{x} s_{y}\right|} \cdot n\left(I_{x}, I_{y}\right) .
\end{gathered}
$$

The factor 4 arises because there are four combinations of $s_{x}, s_{y}$ which give the same values of $I_{x}, I_{y}$. To find the relation between $f\left(I_{x}, I_{y}\right)$ and $n\left(I_{x}, I_{y}\right)$ we must integrate last equality on $q_{x}, q_{y}$ because

$$
\begin{gathered}
f\left(I_{x}, I_{y}\right) \equiv \frac{D N}{D\left(I_{x}, I_{y}\right)}= \\
\int_{0}^{q_{x, \max }} \int_{0}^{q_{y, \max }} \frac{D N}{D\left(q_{x}, q_{y}, I_{x}, I_{y}\right)} d q_{x} d q_{y} .
\end{gathered}
$$

Integrating and taking into account that

$$
\left|s_{x}\right|=\sqrt{I_{x}^{2}-E_{x}^{2} q_{x}^{2}}, \quad\left|s_{y}\right|=\sqrt{I_{y}^{2}-E_{y}^{2} q_{y}^{2}}
$$

we get

$$
f\left(I_{x}, I_{y}\right)=\pi^{2} \frac{I_{x} I_{y}}{E_{x} E_{y}} n\left(I_{x}, I_{y}\right) .
$$

For density in configuration space we have

$$
\varrho(x, y)=\frac{4}{R_{x} R_{y}} \int_{\Omega} \frac{D N}{D\left(q_{x}, q_{y}, I_{x}, I_{y}\right)} d I_{x} d I_{y}
$$

or

$$
\varrho(x, y)=\frac{4}{R_{x} R_{y}} \int_{\Omega} \frac{I_{x} I_{y} n\left(I_{x}, I_{y}\right) d I_{x} d I_{y}}{\sqrt{I_{x}^{2}-E_{x}^{2} q_{x}^{2}} \sqrt{I_{y}^{2}-E_{y}^{2} q_{y}^{2}}} .
$$

Integration domain $\Omega$ is the set defining by the inequalities

$$
I_{x}^{2} \geq E_{x}^{2} q_{x}^{2}, \quad I_{y}^{2} \geq E_{y}^{2} q_{y}^{2}, \quad \frac{I_{x}^{2}}{E_{x}^{2}}+\frac{I_{y}^{2}}{E_{y}^{2}} \leq 1
$$

Substituting the density $f\left(I_{x}, I_{y}\right)$ into the expression (11), one gets

$$
\varrho(x, y)=\frac{4 E_{x} E_{y}}{\pi^{2} R_{x} R_{y}} \int_{\Omega} \frac{f\left(I_{x}, I_{y}\right) d I_{x} d I_{y}}{\sqrt{I_{x}^{2}-E_{x}^{2} q_{x}^{2}} \sqrt{I_{y}^{2}-E_{y}^{2} q_{y}^{2}}} .
$$

Introducing the variables

$$
\tilde{I}_{x}=\sqrt{\left(I_{x} / E_{x}\right)^{2}-q_{x}^{2}}, \quad \tilde{I}_{y}=\sqrt{\left(I_{y} / E_{y}\right)^{2}-q_{y}^{2}},
$$

we can transform the expression (11) to simpler form

$$
\begin{gathered}
\varrho(x, y)=\frac{4 E_{x} E_{y}}{R_{x} R_{y}} \times \\
\int_{\tilde{\Omega}} n\left(E_{x} \sqrt{\tilde{I}_{x}^{2}+q_{x}^{2}}, E_{y} \sqrt{\tilde{I}_{y}^{2}+q_{y}^{2}}\right) d \tilde{I}_{x} d \tilde{I}_{y},
\end{gathered}
$$

where $\tilde{\Omega}$ is determined by the inequalities $\tilde{I}_{x}^{2}+\tilde{I}_{y}^{2} \leq 1-$ $q_{x}^{2}-q_{y}^{2}, \quad \tilde{I}_{x, y} \geq 0$.

From this expression one can obtain particle distributions in configuration space.

\section{EXAMPLES OF DISTRIBUTIONS}

In the simplest case the phase density is constant: $n=n_{0}$. Substituting it to (13) and integrating, we have

$$
\varrho(t, x, y)=\frac{\pi E_{x} E_{y}}{R_{x}(t) R_{y}(t)} n_{0}\left(1-\frac{x^{2}}{R_{x}^{2}(t)}-\frac{y^{2}}{R_{y}^{2}(t)}\right) .
$$

Other simple examples can be easily obtained if we take phase density (or density $f\left(I_{x}, I_{y}\right)$ ) as function of expression $1-I_{x}^{2} / E_{x}^{2}-I_{y}^{2} / E_{y}^{2}$. Let phase density has the form $n\left(I_{x}, I_{y}\right)=n_{0}\left(1-I_{x}^{2} / E_{x}^{2}-I_{y}^{2} / E_{y}^{2}\right)^{p}$. Substituting to (11) we get

$$
\begin{gathered}
\varrho(t, x, y)=\frac{\pi E_{x} E_{y}}{R_{x}(t) R_{y}(t)}\left(1-q_{x}^{2}-q_{y}^{2}\right)^{p+1} \times \\
\int_{0}^{\sqrt{1-q_{x}^{2}-q_{y}^{2}}}\left(1-\frac{\tilde{I}^{2}}{1-q_{x}^{2}-q_{y}^{2}}\right)^{p} \frac{\tilde{I}}{\sqrt{1-q_{x}^{2}-q_{y}^{2}}} \times
\end{gathered}
$$

$d \frac{\tilde{I}}{\sqrt{1-q_{x}^{2}-q_{y}^{2}}}=\frac{\pi E_{x} E_{y}}{(p+1) R_{x}(t) R_{y}(t)}\left(1-q_{x}^{2}-q_{y}^{2}\right)^{p+1}$,

$p \neq-1$, where $\tilde{I}=\sqrt{\tilde{I}_{x}^{2}+\tilde{I}_{y}^{2}}$. For example, if $n$ is proportional to $\left(1-I_{x}^{2} / E_{x}^{2}-I_{y}^{2} / E_{y}^{2}\right)^{-1 / 2}$, then $\varrho$ is proportional to $\left(1-q_{x}^{2}-q_{y}^{2}\right)^{1 / 2}$. In both examples particle density falls down to zero on the border of the beam. It can be seen that for all $p>-1$ this property take place.

An interesting distribution we get taking the density $n\left(I_{x}, I_{y}\right)$ in a form

$$
n\left(I_{x}, I_{y}\right)=n_{0} e^{-\left(\frac{I_{x}^{2}}{E_{x}^{2}}+\frac{I_{y}^{2}}{E_{y}^{2}}\right) / \alpha^{2}} .
$$

Then we have

$$
\begin{gathered}
\varrho(t, x, y)=\frac{\pi E_{x} E_{y}}{R_{x}(t) R_{y}(t)} n_{0} \alpha^{2} \times \\
\left(e^{-\frac{x^{2} / R_{x}^{2}(t)+y^{2} / R_{y}^{2}(t)}{\alpha^{2}}}-e^{\left.-\frac{1}{\alpha^{2}}\right) .}\right.
\end{gathered}
$$

If $\alpha$ is small, then the density is determined by the first term and represents Gauss distribution on transverse coordinates. 


\section{INTEGRAL EQUATION FOR DENSITY}

Another problem of great interest is self-consistent distributions for intense beam. Consider stationary beam in RF electric field, transverse components of which are linear functions of coordinates and have form (1). As above, assume that initially particles fill ellipsoid (2) in phase space of transverse motion, and that in each cross section of the beam all particles have the same longitudinal velocity and fill ellipse (8). Under assumption that particles are uniformly distributed in beam cross section, the particles dynamics equations take the form $[3,4]$

$$
\begin{aligned}
& \frac{d^{2} x}{d t^{2}}=Q_{x} x+\frac{\lambda x}{R_{x}\left(R_{x}+R_{y}\right)}, \\
& \frac{d^{2} y}{d t^{2}}=Q_{y} y+\frac{\lambda y}{R_{y}\left(R_{x}+R_{y}\right)},
\end{aligned}
$$

and the envelope equations are

$$
\begin{aligned}
\frac{d^{2} R_{x}}{d t^{2}} & =Q_{x} R_{x}+\frac{\lambda}{R_{x}+R_{y}}+\frac{E_{x}^{2}}{R_{x}^{3}} \\
\frac{d^{2} R_{y}}{d t^{2}} & =Q_{y} R_{y}+\frac{\lambda}{R_{x}+R_{y}}+\frac{E_{y}^{2}}{R_{y}^{3}}
\end{aligned}
$$

The equations (14), (16) and (15), (17) represent two pairs of systems analogous to the Ermakov system and have the same integrals (6), (7) as systems (3), (4), (5). Moreover, all expressions for densitiy $\varrho(x, y)$ (11-13) obtained above are valid in this case. Taking into account the expression for density (11) we have integral equation for density $f\left(I_{x}, I_{y}\right)$ :

$$
\begin{gathered}
E_{x} E_{y} \int_{\Omega} \frac{f\left(I_{x}, I_{y}\right) d I_{x} d I_{y}}{\sqrt{I_{x}^{2}-E_{x}^{2} q_{x}^{2}} \sqrt{I_{y}^{2}-E_{y}^{2} q_{y}^{2}}}= \\
\pi^{2} R_{x} R_{y} \varrho_{0} .
\end{gathered}
$$

The problem is to find such function $f\left(I_{x}, I_{y}\right)$ that result of the integration is independent on $q_{x}, q_{y}$ (though the integrand is depend on them).

The well known KV distribution $[3,4]$ is the solution of the equation (18):

$$
f=f_{0} I_{x} I_{y} \delta\left(1-I_{x}^{2} / E_{x}^{2}-I_{y}^{2} / E_{y}^{2}\right) .
$$

\section{CONCLUSION}

The approach for determination of particles distributions in electric field proposed in the present report allows modeling of nonuniform distributions for low intensity beams, which can be represented in analytical form along accelerating and focusing channel. In particular, these distributions can be widely used in various optimization problems of beam dynamics with the account of particle density distribution in configuration space $[5,6]$.

Another result of this work is integral equation for density $f\left(I_{x}, I_{y}\right)$ for intense uniform charged beam. On the base of this equation the problem of finding of selfconsistent distributions can be examined.

\section{REFERENCES}

[1] O.I.Drivotin, D.A.Ovsyannikov, "New Classes of Uniform Distributions for Charged Particles in Magnetic Field," PAC'97, Vancouver, B.C., Canada, 1997. Proc. of the 1997 Part. Accel. Conf. Pp.1944-1945.

[2] V.P.Ermakov, Univ.Izv.(Kiev), 20 (9), 1-25 (1880).

[3] I.M.Kapchinsky, Particles Dynamics in Resonant Linear Accelerators Atomizdat, Moscow, 1966.

[4] M.Reiser, Theory and Design of Charged Particle Beams, John Wiley \& Sons, New York, 1994.

[5] D.A.Ovsyannikov, "Mathematical Methods of Optimization of Charged Particles Beam Dynamics," EPAC'96, Sitges(Barcelona), 10-14 June 1996. Proc. of the 5th Europ.Part.Accel.Conf. Vol.2. Pp. 1382-1384.

[6] O.I.Drivotin, D.A.Ovsyannikov, Yu.A.Svistunov, M.F.Vorogushin, "Mathematical Models for Accelerating Structures of Safe Energetical Installation," EPAC'98, Stockholm, 22-26 June 1998. Proc. of the 6th Europ.Part.Accel.Conf. Pp. 1227-1229. 